\title{
Ragni delle Filippine, I. Un nuovo Althepus cavernicolo dell'Isola di Mindanao (Araneae, Ochyroceratidae)
}

di

\author{
Paolo Marcello BRIGNOLI*
}

Tra il numeroso materiale del Museo di Copenaghen da poco affidatomi in istudio dal Dr. S.L. Tuxen e dal Dr. B. Petersen, che vivamente ringrazio, erano anche due Ochyroceratidae raccolti in una grotta di Mindanao nel corso della missione della Noona Dan. Com'era logico attendersi, visto che delle intere Filippine erano note solo due altre specie di questa famiglia, Psiloderces egeria Simon 1892 della grotta di Calapnitan e Theotima microphthalma (Simon) 1892 della grotta di Antipolo (ambedue di Luzon), la specie raccolta dalla missione danese era nuova.

\section{ALTHEPUS Noonadanae N.SP.}

Filippine - Mindanao, distretto di Curuan, grotta di Latuan (località tipica), 21.XII.61, Noona Dan Exp. leg., 2 \% (holo- et paratypus; collez. Zoologisk Museum Kфbenhavn).

DESCRIZIONE - $q$ : colore di fondo del prosoma gialliccio; di bruno violaceo sono colorati il clipeo (specie il margine anteriore e la parte centrale) e la regione oculare come pure il centrc del prosoma, con una banda che giunge al margine posteriore ed i margini laterali del prosoma (queste ultime zone colorate non confluiscono però in nessun punto con quelle del clipeo o della zona centrale). Sei occhi nella tipica disposizione; mediani appena più grandi dei laterali (7:6); occhi mediani accostati tra loro, come pure i laterali; intervallo MA-LA pari alla metà del diametro dei LA. Labium nettamente articolato con lo sterno e più lungo che largo, v.fig. 5: gnatocoxe, v.fig.5. Sterno cordiforme, bruno violaceo salvo una zona chiara a forma di " $\mathrm{T}$ " rovesciata (holotypus) o di trapezio rovesciato (paratypus; N.B.: margine posteriore sempre scuro); mancano tubercoli pronunciati. Cheliceri, v.fig. 4. Palpi, v.fig. 1, piuttosto sottili ed allungati. Zampe annulate di bruno (specie le tibie); tarsi e metà prossimali dei femori giallicci. Opistosoma ovale appuntito; colore di fondo bianchiccio, assai irregolarmente macchiato di bruno-violaceo, sui lati macchie confluenti in strisce parallele; sotto scuro più uniformemente. Vulva, v.fig. 7. Filiere e colulo, v.fig. 2. ot ignoto.

\footnotetext{
* Istituto di Zoologia dell'Università di Roma - Viale dell'Universita 32: 00100-ROMA
} 
DIMENSIONI (in mm, leggermente approssimate) - $q$ holotypus : prosoma lungo 1,07, largo 0,92; opistosoma 2,37. Lunghezza totale : 3,44.

$\begin{array}{llllllr}\text { Zampe } & \text { Femori } & \text { Patelle } & \text { Tibie } & \text { Metatarsi } & \text { Tarsi } & \text { Totale } \\ \text { I } & 3,87 & 0,32 & 4,12 & 4,20 & 1,25 & 13,76 \\ \text { II } & 3,00 & 0,30 & 3,07 & 3,07 & 1,00 & 10,44 \\ \text { III } & 2,25 & 0,30 & 2,00 & 1,82 & 0,80 & 7,17 \\ \text { IV } & 3,45 & 0,30 & 3,37 & 3,25 & 1,00 & 11,37\end{array}$

DERIVATIO NOMINIS: il nome di questa specie deriva da quello della nave su cui è stata effettuata la crociera durante la quale essa è stata raccolta.

DISCUSSIONE: questa è la sesta specie nota del genere Althepus Thorell 1898; delle altre cinque A.pictus Thorell 1898 è della Birmania, A.stellatus (Simon) 1905 è di Giava, $A$. machadoi Brignoli 1973 e $A$. mulcatus Brignoli 1973 sono del Nepal, A.incognitus Brignoli 1973 è dell'India meridionale. Per semplificare l'esame dei

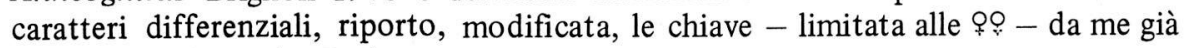
altrove pubblicata (1973a):

1. Margine inferiore dei cheliceri privo di denti $\ldots \ldots \ldots \ldots$. stellatus (Simon)

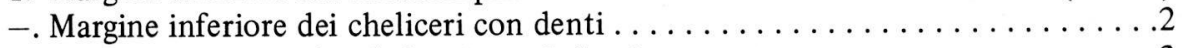

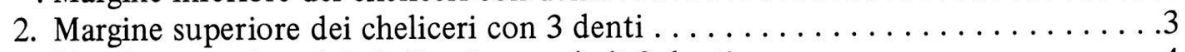

-. Margine superiore dei cheliceri con più di 3 denti $\ldots \ldots \ldots \ldots \ldots \ldots \ldots$

3. 3 denti ben distinti ...................... incognitus Brignoli

-. 2 dei 3 denti corrispondono alle punte della lamella trasparente mediale.......

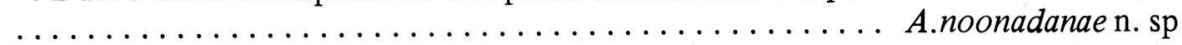

4. Margine superiore dei cheliceri con 5 denti ............mulcatus Brignoli

-. Margine superiore dei cheliceri con 4 denti $\ldots \ldots \ldots \ldots \ldots \ldots \ldots \ldots$

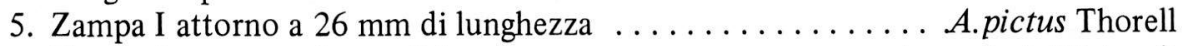

-. Zampa I attorno a $5 \mathrm{~mm}$ di lunghezza ........... machadoi Brignoli

Rispetto alle tre specie da me recentemente descritte (Brignoli, 1973a, 1973b) la nuova specie è anche distinguibile in base alle forma dei genitali delle $\$ \wp$ (ignoti in A.pectus e A. stellatus).

Parlare di affinità è del tutto prematuro, visto che certamente sono ancora assai numerose le specie da descrivere di questo genere del SE asiatico; basti quindi aver sottolineato i caratteri differenziali. Questi stessi caratteri sono infatti del tutto insufficienti per giudicare con quale delle specie già note A.noonadanae presenti reali affinità.

La nuova specie presenta ben poco di peculiare dal punto di vista morfologico; è provvista di polmoni, come sembra la norma negli Ochyroceratidae di maggiori dimensioni; normali sono gli organi liriformi (v.fig. 6) come pure labium e gnatocoxe. Il palpo è piuttosto allungato e può ricordare quello di Psiloderces egeria; interessante è che gli stigmi tracheali posteriori sono esternamente pressochè 


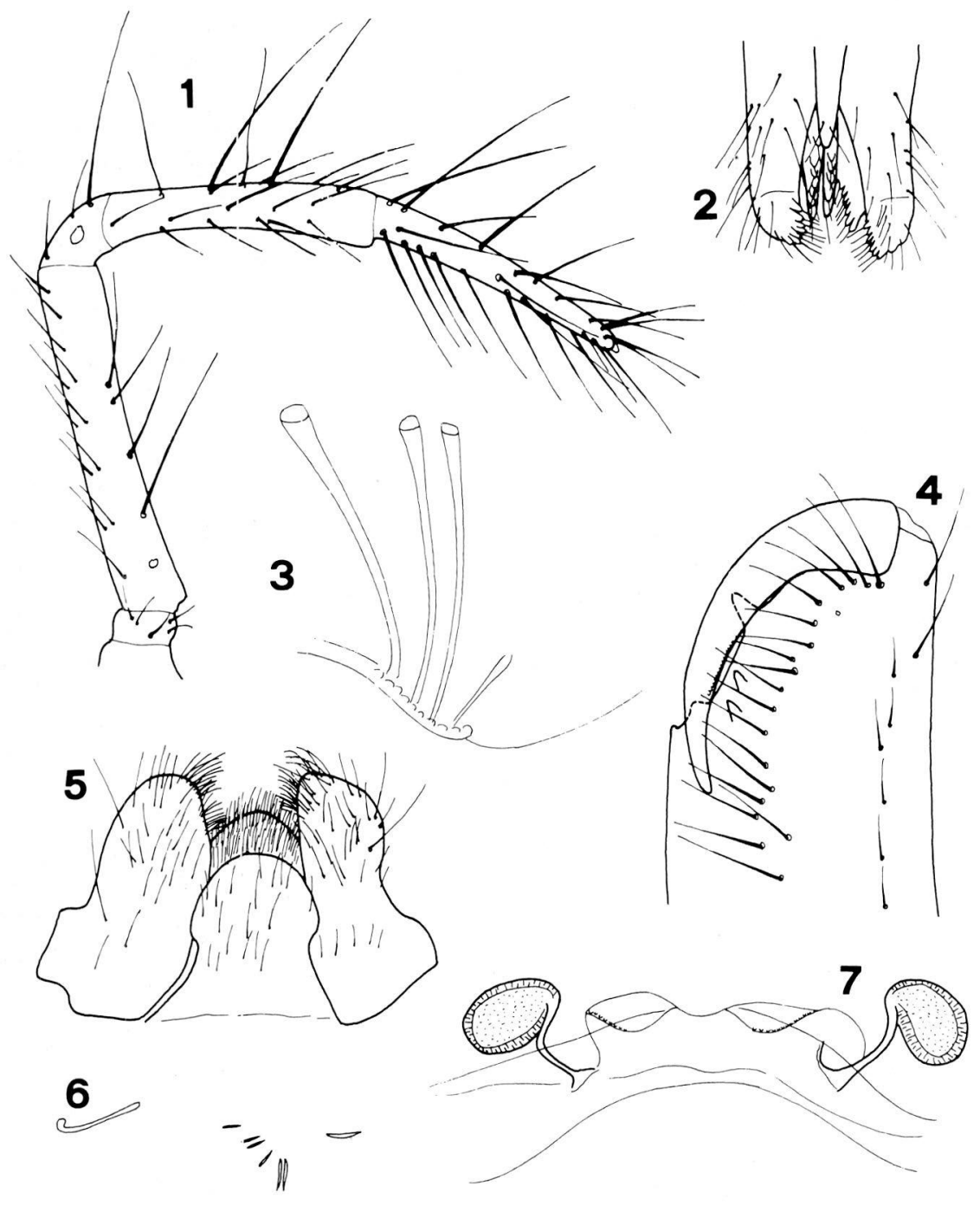

\section{0,05 \\ $1250,3 \mathrm{~mm}, 6 \quad 0,2 \mathrm{~mm}, 4.7 \quad 0,2 \mathrm{~mm}$}

Althepus noonadanae n.sp. - Fig. 1: palpo della 우 fig. 2: filiere e colulo; fig. 3: tubuli annessi alla vulva; fig. 4; chelicero; fig. 5: labium e gnatocoxe (asimmetria dovuta al preparato); fig. 6: stigma polmonare destro ed organi liriformi (da sotto; fig. 7: vulva. 
separati (sono però comunicanti internamente). Da una della lamella ripiegate che circondano le spermateche si dipartivano dei singolari tubuli, di ignoto significato (v.fig. 3).

Con l'occasione debbo far notare che è assai verosimile che i generi Althepus Thorell, Merizocera Fage e Psiloderces Simon debbano essere ridefiniti, sulla base di caratteri più validi (genitali); sfortunatamente di molte specie i ớ sono ancora ignoti e mancano descrizioni dei genitali femminili.

Questa specie è la quinta della famiglia trovata in una grotta del SE asiatico; a parte infatti Psiloderces egeria e Theotima microphthalma, Althepus pictus fu anche citato delle Farm Caves in Birmania e Psiloderces crinitus Fage 1929 è stato descritto delle Batu Caves in Malesia. A proposito di quest'ultima specie, è da notare che, in base al ò scoperto da Bristowe (1952), la sua posizione generica non è più del tutto chiara; il palpo del ô infatti, ricorda assai quello di Merizocera cruciata (Simon), come illustrato da Fage (1912).

Althepus noonadanae n.sp. non presenta alcun adattamento alla vita cavernicola; è quindi al più solo un troglofilo, di un gruppo però ormai da considerare tipico delle grotte tropicali, forse l'equivalente dei Leptonetidae delle grotte temperate.

Sfortunatamente la fauna delle grotte delle Filippine, che deve essere di estremo interesse, a giudicare dai pochi dati esistenti (ricordo solo la "migale" cieca Accola caeca Simon ed i Blattoidei cavernicoli del genere Nocticola Bolivar), è ancora pressochè sconosciuta.

\section{RIASSUNTO}

Viene descritto Althepus noonadanae n.sp. ( $\$$; ${ }^{*}$ ignoto; loc. typ.: grotta di Latuan, distretto di Curuan, Mindanao, Filippine); distinguibile dalle altre specie note per la morfologia dei cheliceri e dei genitali della $\$$. Per il momento è impossibile avvicinare questa specie a nessuna di quelle già note. Viene proposta una chiave per le $\$$ di Althepus.

\section{SUMMARY}

Althepus noonadanae n.sp. is described ( $\%$; ${ }^{\star}$ unknown; loc. typ.: Latuan Cave, Curuan district, Mindanao, Philippines); it can be distinguished from the other known species by the morphology of the chelicerae and of the $q$ genitalia. It is not related to any of the very few described species. A key for the $q$ Althepus is given.

\section{BIBLIOGRAFIA}

BRIGNOLI, P.M., 1973a - Un nuovo Althepus dell'India meridionale. Rev. Suisse Zool., (in stampa).

BRIGNOLI, P.M., 1973b - Spinnen aus Nepal, II. Zur Morphologie der Gattung Althepus, nebst Beschreibungen zwei neuer Arten. Senckenberg. biol., (in stampa). 
BRISTOWE, W.S., 1952 - The Arachnid fauna of the Batu Caves in Malaya. Ann. Mag. Nat. Hist., (12) $5:$ : 697-707.

FAGE, L., 1912 - Etudes sur les araignées cavernicoles. I. Révision des Ochyroceratidae (n.fam.). Arch. Zool. Expér. Génér., (5) 10 (Biospeologica 25) : 97-162.

FAGE, L., 1929 - Fauna of the Batu Caves, Selangor. X. Arachnida. Journ. Fed. Malay St. Mus., 14 (3-4) : 356-364. 